\title{
EXPERIMENT ON A STABLE MOTION OF THE BACKHOE EXCAVATOR BUCKET
}

\author{
E. Budny, M. Chłosta, W. Gutkowski \\ mch@imbigs.org.pl
}

Institute of Mechanised Construction and Rock Mining, Warsaw, Poland

\begin{abstract}
1. INTRODUCTION
Recently, there is an increasing interest in controlled excavation processes. The modern control software and hardware make hydraulic systems more suitable for enhancement of a large spectrum of human efforts. This is mainly through control of repetitive processes, such as trenching and drilling, requiring constant attention of machine operators. The basic attention, in research, is paid to excavation along prescribed trajectories subjected to varying soil environment. Fundamentals dealing with controlled excavation processes are discussed by Hemani [4], Hiller and Schnider [5]. An interesting approach to piling processes by a direct angular sensing method is proposed by Keskinen et al. Budny and Gutkowski $[1,2]$ propose an open loop system, applying load - independent hydraulic valves. In this case, influence of small variation of hydraulic oils into actuators is discussed by Gutkowski and Chlosta [3], applying sensitivity analysis. Huang et al. [6] presented an impedance control study for a robotic excavator. They applied two neural networks. One as a feed forward controller, and the second one as a feedback target impedance.

The first generation of robots were conceived as 'open loop" positioning devices. This implied that all parts had to be manufactured with a very high and costly accuracy. Next positioning robots, with sensors, reduced this accuracy requirement considerably.

Here were several approaches, mentioned in above references, to extend the industrial robots capabilities to robotic excavator. System of force cells, longitudinal and angular sensors have been applied. However, two main differences between requirements for industrial robots and robotic excavators should be noted. The first one is that industrial robots are working in almost perfect conditions, free of vibrations, protected against shocks, humidity and other possible damaging conditions. The second one is requirements for their high accuracy, often within microns. On the contrary, robotic excavators are working in very heavy conditions, and required accuracy of the executed trajectories, comparing with industrial robots, is limited, say within centimetres. With very heavy conditions of excavations works, all
\end{abstract}

sensors attached to the boom, arm and bucket have to be very well protected.

Bearing in mind above differences it would be of interest to investigate the possibilities of controlling excavation trajectory by hydraulic module composed of a pump and loadindependent valves. Other words, to investigate an "open loop" system free of sensor cells mounted at the excavator attachment, combined with a feedback controller included in the hydraulic unit of the machine. The main objective of the present paper is then to propose such a control system for controlled excavation along prescribed path.

\section{STATEMENT OF THE PROBLEM}

The paper deals with a controlled, stable motion of an excavator bucket along a prescribed path. The problem based on previous authors' theoretical investigations of quasi-static, kinematically induced excavation processes for assumed trajectories. In this study the following assumptions are made:

- It is assumed that the excavator attachment is a planar mechanism, composed of two arms and a bucket. Three independently driven, hydraulic actuators, operates the system. They are assuring a unique representation of the three degrees of the planar bucket motion, two displacements of the bucket tip and its rotation.

- The excavation process is assumed quasi static, and then inertia terms in motion equations of attachment can be neglected.

- The force (pressure) disturbances, from assumed pressure, are assumed to have sinusoidal form. The acceptable parameters of the sinusoid are defined from stability conditions of the system.

- The soil is assumed homogeneous, some small inclusions in the form of stone.

- The proposed control system of excavation is operator assisted. It means that in cases of larger obstacles the operator has to intervene.

- If successful, the proposed control setup could apply to standard excavators with the aim of enhancement of a large spectrum of 
human efforts in repetitive processes such as trenching and drilling.

- The experiment is considered as a dynamic system composed of three subsystems, namely: PC with PLC; hydraulic arrangement (a pomp, valves, actuators); and the mechanism with the bucket with three degrees of freedom. Next, the subsystems are considered as sets of components. In the first subsystem, the following components are recognised: personal computer with appropriate software, transforming introduced equations and inequalities of motion and trajectory planers into electric signal. The latter is send to a PLC unit, which in turn causes an electrical actuation of solenoid valves. Pressures from these valves are causing change of spool position, assuring flow of hydraulic oil into actuators. The spool position, in turn, is converted, through a transducer sending an electric feedback signal to the solenoid valves. Opened spools are sending the hydraulic oil into the third subsystem, namely actuators of the excavator mechanism. Finally, the last subsystem is composed of three components: hydraulic actuators; arms; and the bucket. With the motion of the excavator, arms and the bucket itself, the pressures in actuators are changing. Information about these changes is sent to the second, hydraulic subsystem, where the feedback signal corrects position of spools assuring the oil flow according to the designed trajectory.

In the paper, transfer functions of all system components are investigated from the point of view of stability. The functions are defined theoretically or experimentally from diagrams presented in catalogues of hydraulic equipment. Joining together all transfer function of particular component, the transfer function of the whole system is discussed from the point of view of stability.

Several experiments were performed, showing that it is possible to assure stable, assumed motion of the bucket. Among experiments one was devoted to drilling. In the other words, the kinematically induced trajectory was a straight, vertical line. Experimentally obtained line is presented in Fig. 3 and Fig. 4. It is interesting to note that variation of experimental line doesn't exceed $10 \mathrm{~cm}$.

\section{THREE SUBSYSTEMS OF THE EXPERIMENTAL SETUP}

The discussed system is divided in three subsystems, namely: PC, hydraulic valves, and excavator arms with a bucket. Below, they are discussed separately, and then a joint transfer function (TF) is defined.

\subsection{PC AS A SUBSYSTEM}

We start with defining a model of the bucket motion. The bucket, it its plane motion, has three degrees of freedom $\alpha_{j}(\mathrm{j}=1,2,3)$ (Fig. 1). They are relative rotations of the boom with respect to the excavator body, of the arm with respect to the boom, and the bucket with respect to the arm. Angular velocities, related to these rotations, are denoted by $\boldsymbol{\omega}$. The bucket itself has also three degrees of freedom, in a planer motion here considered.

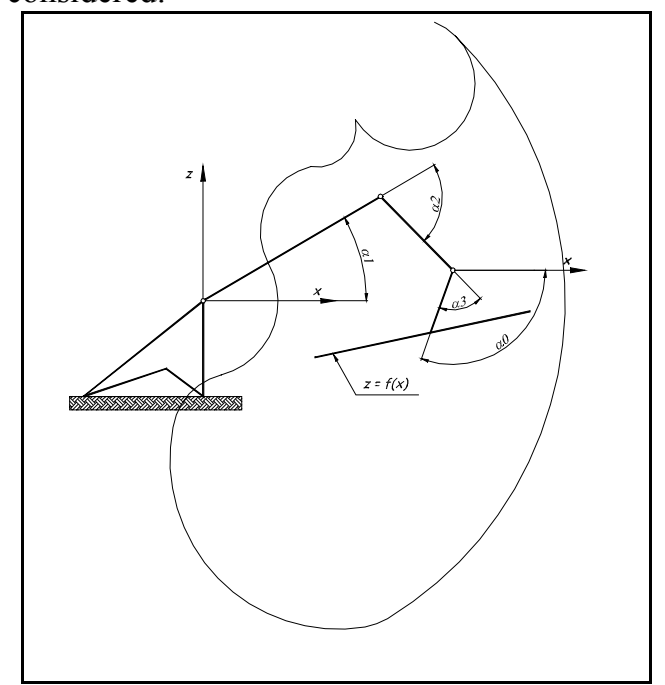

Fig. 1. Miniexcavator with its attachment.

They are, two components $\boldsymbol{v}_{1}$ and $\boldsymbol{v}_{2}$ of the tip velocity along $\boldsymbol{x}$ and $\boldsymbol{z}$ axes, and its angular velocity $\boldsymbol{v}_{3}$. Its velocity vector $\mathbf{v}$ is then equal to

$v=\lfloor\dot{x}, \dot{z}, \dot{\theta}]=\left[v_{1}, v_{2}, v_{3}\right]$

Denoting now the angular velocity vector as

$\omega=\left[\dot{\alpha}_{1}, \dot{\alpha}_{2}, \dot{\alpha}_{3}\right]=\left[\varpi_{1}, \varpi_{2}, \varpi_{3}\right]$

The relation between $\boldsymbol{v}$ and $\boldsymbol{\omega}$ is given by

$v=A \cdot \omega$

Where

$$
\begin{aligned}
& A_{11}=-l_{1} \sin \alpha_{1}-l_{2} \sin \left(\alpha_{1}+\alpha_{2}\right)-l_{3} \sin \theta \\
& A_{12}=-l_{2} \sin \left(\alpha_{1}+\alpha_{2}\right)-l_{3} \sin \theta \\
& A_{13}=-l_{3} \sin \theta \\
& A_{21}=l_{1} \cos \alpha_{1}+l_{2} \cos \left(\alpha_{1}+\alpha_{2}\right)+l_{3} \cos \theta \\
& A_{22}=l_{2} \cos \left(\alpha_{1}+\alpha\right)+l_{3} \cos \theta \\
& A_{23}=l_{3} \cos \theta \\
& A_{31}=A_{32}=A_{33}=1
\end{aligned}
$$


Angular velocities $\omega_{j}$ are in turn dependent on the elongation velocities $\boldsymbol{w}_{\boldsymbol{j}}$ of hydraulic actuators. Their relation has the following form

$$
W=H \omega
$$

where $\boldsymbol{H}$ is a $3 \times 3$ matrix with components, which are defined applying geometrical consideration given in $[1,2]$. The velocities $\boldsymbol{\omega}$ are now dependent on the oil flow $\boldsymbol{q}_{j}$ into actuators. This dependence can be presented again with $3 \times 3$ matrix as

$q=S \cdot \omega$

The $\boldsymbol{S}$ is a diagonal matrix with components equal to the cross section areas of actuator pistons.

Combining equations (1) to (6) we get the following relation between the oil flow into actuators and three velocities of the excavator backet

$$
Q=S \cdot H \cdot A^{-1} \cdot v
$$

These values are now converted into electric signal, which activate the hydraulic valve.

\subsection{HYDRAULIC VALVE SYSTEM \\ (HVS)}

The PC information, through PLC is converted into an electric signal to the valve. The HVS is presented in Fig. 2. Bellow we discuss all its parts and their transfer functions.

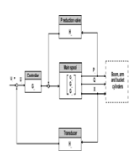

Fig. 2. HSV diagram.

The difference between the references signal $\boldsymbol{u}_{\boldsymbol{i}}$ and a signal $\boldsymbol{u}_{\boldsymbol{d}}$ from feedback, is activating the controller. Denoting by capital letters Laplace's transforms of variables, we find:

$$
\begin{aligned}
U_{C}(s) & =U_{i}(s)-U_{d}(s)= \\
& =U_{i}(s)-H_{U X}(s) X_{S}(s)
\end{aligned}
$$

where $\boldsymbol{X}_{\boldsymbol{s}}(\boldsymbol{s})$ is main spool displacement, and $\boldsymbol{H}_{\boldsymbol{u} \boldsymbol{x}}$ is a transfer function between spool displacement and $\boldsymbol{U}_{\boldsymbol{d}}$ signal. The latter is obtained by a transducer, with constant multiplier, giving transfer function $\boldsymbol{H}_{\boldsymbol{u} \boldsymbol{x}}$ :

$$
H_{U X}=U_{d}(s) / X_{S}=k_{d}
$$

Also, relation between $\boldsymbol{U}_{\boldsymbol{C}}$ and $\boldsymbol{p}_{\boldsymbol{C}}$, coming out from the controller is constant and the transfer function is:

$G_{p u}(s)=P_{C}(s) / V_{C}(s)=k_{u}$

Next, the $\boldsymbol{P}_{\boldsymbol{C}}(\mathbf{s})$ pressure is added to the $\boldsymbol{P}_{\boldsymbol{d}}$ pressure caused by disturbances and it is acting on the main spool. The Laplace's transform of the spool motion equations is as follows:

$$
\left(s^{2} M_{S}+s C_{S}+k_{S}\right) \cdot X_{S}(s)=P_{S}(s) \cdot A_{S}
$$

where: $\mathrm{M}_{\mathrm{S}}, \mathrm{C}_{\mathrm{S}}, \mathrm{k}_{\mathrm{S}}$, and $\mathrm{k}_{\mathrm{S}}$; are :

- $\quad$ the spool mass $[\mathrm{kg}]$;

- damping coefficient;

- $\quad$ spring constant;

- and the cross section area on which the pressure is acting.

The transfer function between displacement and pressure is:

$$
\begin{aligned}
G_{x p} & =X_{S}(s) / P_{S}(s)= \\
& =A_{S}\left(s^{2} M_{S}+s C_{S}+k_{S}\right)
\end{aligned}
$$

The volumetric fluid flow rate $q\left(x_{s}, p_{s}\right)$ is a function of displacement $\boldsymbol{x}_{\boldsymbol{s}}$ and effective pressure $\boldsymbol{p}_{\boldsymbol{e}}$ in the valve actuator. Applying the Taylor series linearization we get:

$$
\begin{aligned}
q\left(x_{s}, p_{e}\right) & =\left(\frac{\partial q}{\partial x_{s}}\right)_{x_{0} p_{e 0}} x+\left(\frac{\partial q}{\partial p_{e}}\right)_{x_{0} p_{e 0}} p_{e}= \\
& =k_{q} x_{s}-k_{p} p_{e}
\end{aligned}
$$

where $\boldsymbol{p}_{\boldsymbol{e}}$ is an effective pressure equal to the sum of reference pump pressure and disturbance pressure caused by varying excavator attachment load. It is assumed that the disturbance pressure $\boldsymbol{p}_{\boldsymbol{d}}$ can take a sinusoidal form: 
$p_{d}(t)=\Delta p_{0} \cdot \sin X t$

in which the $\Delta \boldsymbol{p}_{0}$ and $\boldsymbol{X}$ have to be assumed for the given process. The Laplace transformation of (14) is:

$$
p_{d}(s)=\Delta p_{0} \frac{X}{s^{2}+X^{2}}
$$

The relation between pressure $\boldsymbol{p}_{\boldsymbol{s}}(\mathbf{s})$ and $\boldsymbol{q}_{\boldsymbol{s}}(\mathbf{s})$ is given by specification of applied valve. In our experiment it is the PVG 32 type Danfoss loadindependent proportional valve. The diagram $\boldsymbol{p}_{\boldsymbol{s}}(\boldsymbol{s})$ versus $\boldsymbol{q}_{\boldsymbol{s}}(\boldsymbol{s})$ shown in the manufacture catalogue was linearized. The Laplace transform of the transfer function $\boldsymbol{G}_{\boldsymbol{q}}$ is equal to:

$G_{q p}(s)=\frac{Q(s)}{p_{s}(s)}=k_{p}$

After standard transformation we get the Laplace's transform of the oil flow $Q(s)$ :

$Q(s)=\left[U_{i}(s) \cdot k_{n}+p_{d}(s)\right] \cdot \frac{k_{p}\left(s^{2} M_{s}+s C_{s}+k_{s}\right)}{s^{2} M_{s}+s C_{s}+\left(k_{s}+A_{s} k_{n} k_{d}\right)}$

We observe that all the coefficients of the denominator polynominal have the same sign. The necessary condition for the stability of the close-loop system is then fulfilled. Other condition for stable system can be verified only by substituting for particular coefficient their numerical values.

\section{EXPERIMENTAL RESULTS.}

To examine the performance of the proposed control system a mini excavator with three hydraulic cylinders and then tree degrees of freedom is used. An electro-hydraulic, load independent, proportional valve controls each cylinder.

Experiments have been done in order to control the motion of the bucket tip along prescribed path. The motion was controlled both in free space and soil box, filled with homogenous mildly humid sand.

The first experiment was performed with relatively small velocity of about $2 \mathrm{~m} / \mathrm{min}$. Lines obtained for drilling along a vertical and horizontal line is presented in Fig. 3 and Fig. 4.

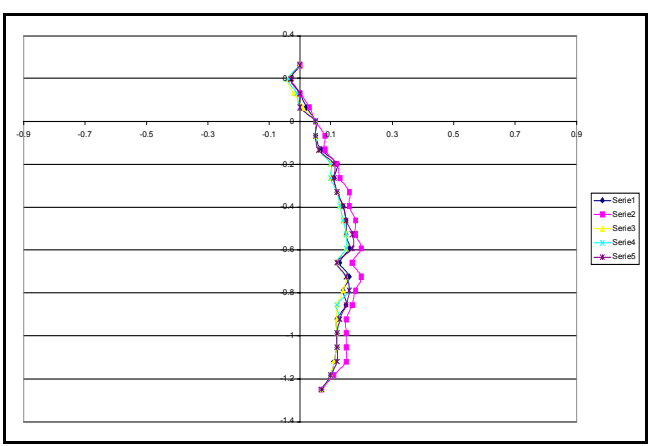

Fig. 3.Experimental trajectory along vertical line

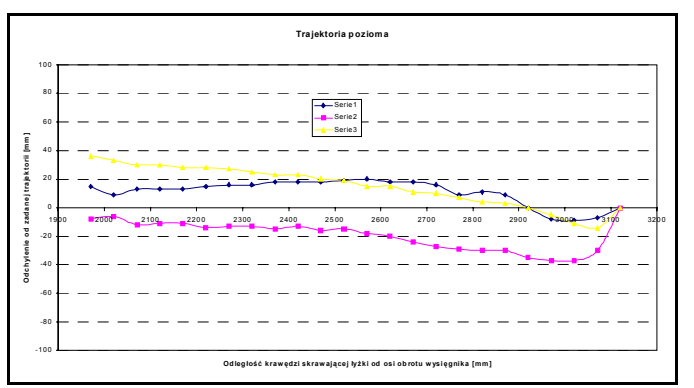

Fig. 4. Trajectory in the horizontal movement.

\section{CONCLUSIONS}

The paper presents theoretical and experimental results on controlled excavation along prescribed trajectories. The assumed path of a bucket tip is introduced into PC. The computer is sending commands to a hydraulic system of three loadindependent valves. This way the assumed trajectory can be executed independently of variations of digging forces, gravity forces of excavator attachment elements, and the soil filled bucket. The trajectory is then executed without usually applied multisensor systems with load cells and angular or longitudinal encoders.

Investigating transfer functions of all elements of the controlled system, it was possible to avoid an unstable motion of the bucket. Obtained results, for three different trajectories, are showing that the present method gives relatively not expensive system of controlled excavation. It could be especially useful for repetitive operations under surveillance of machine operators.

\section{REFERENCES}

[1] Budny E., and Gutkowski W., Kinematically induced excavation by backhoe excavator, 13 th ISARC, Proceedings, 1996, pp 673-680

[2] Budny E., Gutkowski W., A hydraulic open loop system for controlled excavation along prescribed path.

[3] Gutkowski W., Chłosta M., Sensitivity of the Bucket Motion in Controlled Excavation, ANC 8 th Int. Topical Meeting on Robotics and Remote Systems, April 25-29, 1999, Pittsburgh 
[4] Hemani A., Fundamental analysis of automatic excavation, J. Aerospace Eng. Oct. 1995, vol.8, No 4, pp 175-179

[5] Hilles M., Schnider M., Modeling, simulation and control of flexible manipulators, Eur. J. Mech. A/Solid vol.16, 1997, Special Issue, pp 127-150

[6]HuangX.,BernoldL.,LeeG.,(1996) A neural network impedance learning control model for a robotic excavator,ASCE Speciality

Conference on Robotics for Challenging Enviroments, Albuquerque, New Mexico, June 1-6 pp. 213-219

[7] Keskinen E., Launis S., Cotsafis M., Raunisto Y., Trajectory Control Performance Analysis of Excavator - Based Sheet - Piles System

[8] Vaha P.K., Skibniewski M.J., Cognitive force control of excavators, J. Aero. Eng. ASCE vol.6, No 2, 1993 